\title{
Impact of sunitinib-induced hypothyroidism on survival of patients with metastatic renal cancer
}

Theofanis Vasileiadis ${ }^{1,5^{*}}$, Michail Chrisofos ${ }^{2}$, Michail Safioleas ${ }^{3}$, Konstantinos Kontzoglou ${ }^{4}$, Konstantinos Papazisis ${ }^{1,6}$ and Athina Sdrolia ${ }^{1,7}$

\begin{abstract}
Background: Sunitinib plays an important role in managing the metastatic renal cell cancer (mRCC). Sunitinibinduced hypothyroidism is a common side-effect of the drug. There have been attempts to link hypothyroidism with a better clinical outcome in sunitinib-treated (mRCC) patients. Our aim was to relate the impact of hypothyroidism to the survival of these patients.

Methods: We have evaluated 70 patients with mRCC that received sunitinib as a first line treatment. Thyroid-stimulating hormone (TSH) was measured at baseline, after 15 days of treatment (day-15) and at the end of the second cycle (day-75). Biomarker data and correlations with response were analysed with Microsoft Excel. Comparison results from Student's ttest with a p less than 0.05 were considered statistically significant. Kaplan-Meyer and log-rank tests were performed using GraphPad Prism 5 for Windows.

Results: Regarding the response to treatment, a progression-free survival (PFS) of 9.47 months and an overall survival (OS) of 22.03 months were demonstrated. Our data are consistent with published data by other authors.

On day-15 from the beginning of the treatment an important number of patients exhibited a TSH elevation. On day-15 42.86\% had a TSH over the upper normal limit and 50.0\% at the end of the second cycle (day-75).

TSH increased earlier in patients that exhibited an objective response $(\times 3.33$ times the baseline values on day-15) than patients that exhibited disease stabilisation $(\times 2.18)$ or disease progression $(\times 1.59)$. Early increases in TSH were associated with a longer PFS (11.92 vs. 8.82 months, $p=0.0476)$ and a longer OS (3.10 vs. 1.08 years, $p=0.0011)$.

Conclusions: Early TSH-increase is associated with a clinical benefit. The patients that showed at least a twofold increase of their baseline TSH, responded to therapy by stabilisation or by regression of disease.

This is the only study to our knowledge which shows that early increases - 2 weeks from starting the treatment - in TSH levels have a prognostic value. Both PFS and OS of the patients who demonstrated a higher than a twofold rise were significantly longer than the PFS and the OS of the patients that presented a lower or no TSH-increase.
\end{abstract}

Keywords: Sunitinib, Hypothyroidism, Metastatic renal cell cancer, Clear cell carcinoma, Thyroid-stimulating hormone, TSH

\footnotetext{
* Correspondence: th_vasileiadis@yahoo.gr

${ }^{1}$ Theagenion Cancer Hospital, Al. Symeonidi 2, 54007 Thessaloniki, Greece

${ }^{5}$ Present Address: Endocrinology Department, Hull Royal Infirmary, Anlaby

Road, Hull HU3 2JZ, UK

Full list of author information is available at the end of the article
}

(c) The Author(s). 2019 Open Access This article is distributed under the terms of the Creative Commons Attribution 4.0 International License (http://creativecommons.org/licenses/by/4.0/), which permits unrestricted use, distribution, and reproduction in any medium, provided you give appropriate credit to the original author(s) and the source, provide a link to the Creative Commons license, and indicate if changes were made. The Creative Commons Public Domain Dedication waiver (http://creativecommons.org/publicdomain/zero/1.0/) applies to the data made available in this article, unless otherwise stated. 


\section{Background}

Malignant tumours of the kidney account for more than $3 \%$ of cancer incidents and more than $2 \%$ of cancer mortality in the European Union as estimated in 2014 [1]. In addition, during the last years, the incidence of kidney malignant tumours appears to have an increasing trend [2]. Renal cell carcinomas, representing more than $80 \%$ of all kidney tumours [3], have been treated by means of chemotherapy, interleukin 2 and pegylated interferon alfa- $2 \mathrm{~b}$ insufficiently, with response rates of 6-, 7- and $14 \%$ respectively [4-6]. New agents such as tyrosine kinase inhibitors (TKIs), have initiated a revolution in the treatment of metastatic renal cell carcinoma (mRCC) since their approval in 2006.

The results of a randomised, phase III trial comparing sunitinib with interferon (IFN)-a in treatment-naïve mRCC patients showed a statistically significant higher objective response rate $(31 \%$ vs. $6 \%, p<0.001)$ and a longer progression-free survival (11 vs. 5 months), with a hazard ratio of $0.42(0.32-0.54, p<0.001)$ [7]. However, success did not come without cost; treatment with sunitinib appeared to result in several side effects with hypothyroidism being one of the most common ones [8].

Sunitinib-induced hypothyroidism was first reported in patients with gastrointestinal stromal tumour (GIST) [9], followed by the report of Schoeffski et al referring to both patients with RCC and GIST [10] and by the report of Rini et al referring to patients with RCC [11]. The retrospective study of Rini et al demonstrates that hypothyroidism is caused by sunitinib in $85 \%$ of patients [11]. The prospective study of Wolter et al showed that only $34 \%$ of patients had no biochemical thyroid abnormality [12] while the prospective study of Baldazzi et al exhibited at least one elevated thyroid-stimulating hormone (TSH) in 59\% of patients [13].

Various mechanisms such as the inhibition of thyroid peroxidase activity [14], the block of iodine uptake [due to a direct effect of sunitinib on sodium iodide symporter (NIS)], the block of TSH receptor [15], and alterations in thyroxine/triiodothyronine $\left(\mathrm{T}_{4} / \mathrm{T}_{3}\right)$ metabolism (related to increased activity of type 3 Deiodinase) [16] have been proposed as hypothyroidism-inducing. Cases of atrophy and reduction of the thyroid gland size [17-19], degeneration of the follicular epithelial cells [17] and reduced vascularity [18] have also been related to hypothyroidism. Extra-thyroidal mechanisms may also be involved since there is a reported case of a patient treated with sunitinib who underwent thyroidectomy and developed hypothyroidism [13]. The interference of the hypothalamic-pituitary-thyroid axis is considered unrelated to hypothyroidism since no other relevant hormone abnormalities have been recorded in such cases $[13,15]$.

Several studies suggest that hypothyroidism is associated with cell growth inhibition and a better prognosis of different types of tumours [20-22]. An analogous correlation has been attempted between the sunitinib-induced hypothyroidism of patients with $\mathrm{mRCC}$ and their clinical outcome. The first report referring to this correlation was published by Wolter et al in 2002. The hypothyroid patients appeared with both longer median progression-free survival (PFS) (10.3 vs. 3.6 months) and overall survival (OS) (18.2 vs. 6.6 months) than the respective PFS and OS of patients who remained euthyroid during their treatment [12]. These results are in good agreement with the median PFS reported by Baldazzi et al. ( 8.55 months for hypothyroid patients vs. 7.03 months for euthyroid patients) [13]. In the study of Schmidinger et al focusing on mRCC patients under sunitinib or sorafenib, the rate of objective remission was significantly higher in the hypothyroid patients than in the euthyroid patients $(28.3 \%$ vs. 3.3\%) and the median duration of survival was longer (not reached vs. 13.9 months) [23]. Another study, by Riesenbeck et al, dealing with $\mathrm{mRCC}$ patients under sunitinib or sorafenib showed that a longer median PFS was related to patients who developed hypothyroidism in comparison with the euthyroid patients (16.0 vs. 6.0 months) [24]. Shinohara et al associated the high thyroid volume reduction - accompanied by hypothyroidism - in mRCC patients under sunitinib with a longer medium PFS, as opposed to the low thyroid volume reduction associated more often with euthyroidism [17]. Development of hypothyroidism was used as a prognostic parameter in the study of Sella et al as well. According to this study, hypothyroid mRCC patients under sunitinib treatment tended to have longer median PFS (12.2 vs. 9.4 months) and longer OS (22.4 vs. 13.9 months) than euthyroid patients. Both groups appeared to have similar clinical benefit [25]. Nevertheless, the prospective study of Sabatier et al reported no association between mean PFS and hypothyroidism [26].

We evaluated the impact of hypothyroidism and early changes in TSH levels in the treatment outcome of 70 mRCC patients treated with sunitinib.

\section{Methods \\ Eligibility criteria}

Seventy patients with histologically confirmed metastatic clear-cell RCC were enrolled in our retrospective study. They were consecutive patients as appeared on the database of our Renal Cancer Clinics. Based upon the Eastern Cooperative Oncology Group (ECOG) criteria, all patients had a performance status of $\leq 2$ and they were aged between 18 and 80 years old. For these patients, sunitinib was used as first line therapy. All patients had adequate bone marrow (Haemoglobin $\geq 10.0 \mathrm{~g} / \mathrm{dL}$, White Blood Count $\geq 3000 \times 10^{9} / \mathrm{L}$, Neutrophils $\geq 1000 \times 10^{9} / \mathrm{L}$ and Platelets $\geq 100,000 / \mathrm{mcL}$ ), renal (Creatinine $\leq 2 \mathrm{mg} / \mathrm{dL}$ ), hepatic (transaminases $\leq 3$ times the upper limit of normal values, total bilirubin $\leq 2$ times the maximum limit of normal limit) 
and cardiac (Left Ventricular Ejection Fraction at least $50 \%$ ) function. Patients receiving any medication known to intervene with thyroid function or who had received external neck irradiation or radioiodine therapy were excluded from the analysis.

\section{Drug administration}

Sunitinib was administered in accordance with the conventional 6-week schedule which consisted of 4 weeks of daily administration of $50 \mathrm{mg}$, followed by a 2-week off-treatment interval. Dose reduction $(37.5 \mathrm{mg}$ with the same 4-weeks on and 2-weeks off schedule) was allowed according to the side-effect profile. The patients have been treated with sunitinib from the day of diagnosis until the date of radiologically confirmed relapse or the date they dropped out due to side effects.

\section{Examinations on treatment}

Physical examination, performance status, blood cell counts and serum chemistry were assessed at baseline, on day $-15,-30,-45,-60,-75,-90$ as well as at the beginning and end of the following treatment cycles thereafter. The National Cancer Institute Common Terminology Criteria for Adverse Events version 3.0 were utilised to grade both adverse effects and abnormal laboratory values. Tumour evaluation was performed by means of computed tomography or magnetic resonance imaging as well as bone scintigraphy where needed before the start of sunitinib therapy and at the end of every two cycles. Clinical outcome was assessed by $\mathrm{CT}$ and MRI scans, and reported using the RECIST criteria [27].

\section{Evaluation of thyroid function}

The thyroid function of all patients was appraised at baseline and on days -1 and -28 of each treatment cycle by means of TSH and free $\mathrm{T}_{4}$. Notably at baseline, on day-15 of the first cycle and on day- 28 of the second cycle a number of parameters were evaluated; TSH (reference range $0.30-4.0 \mathrm{mU} / \mathrm{L}$ ), free $\mathrm{T}_{4}$ (reference range $7.8-19.4 \mathrm{pg} / \mathrm{mL}$ ), antibodies against thyroid peroxidase (TPOAb; reference range $\leq 50 \mathrm{U} / \mathrm{mL}$ ), antibodies against thyroglobulin ( $\mathrm{TgAb} ; \leq 70 \mathrm{U} / \mathrm{mL}$ ) and antibodies against the TSH receptor (TR-Ab; reference classification as "negative" when $<9 \mathrm{U} / \mathrm{L}$, "questionable positive" when 9-14 U/L, "positive" when >14 U/L). All serum samples were assayed by immunoradiometric (IRMA) methods and tests were performed in duplicate. Specifically, for TSH and free $\mathrm{T}_{4}$, kits by DiaSorin, Saluggia, Italy were used, while for TPOAb, TgAb and TR-Ab, kits by ZenTech, Angleur, Belgium were used.

According to the guidelines of the American Association of Clinical Endocrinologists and the American Thyroid Association, subclinical hypothyroidism is connected with a serum TSH above the upper reference limit combined with a free $T_{4}$ into the normal reference range, while overt hypothyroidism is characterised by an elevated TSH in combination with a subnormal free $\mathrm{T}_{4}$.

Thyroid replacement with levothyroxine was initiated at the discretion of the physician, based on both serum TSH measurements and any clinically significant symptoms related to hypothyroidism such as fatigue, cold intolerance, weight gain and constipation. The aim of the thyroid replacement was a TSH serum concentration between 0.50 and $2.50 \mathrm{mU} / \mathrm{L}$.

\section{Bioanalytics}

The blood sample collection was followed by their transport on ice and centrifugation in order to separate serum. The storage of the aliquots was carried out at $-80^{\circ} \mathrm{C}$ and they were thawed only once.

\section{Statistical analysis}

Biomarker data and correlations with response were analysed with Microsoft Excel. Comparison results from Student's t-test with a $p$ value less than 0.05 were considered as statistically significant. Kaplan-Meyer and log-rank tests were performed using GraphPad Prism 5 for Windows.

\section{Results}

\section{Patient characteristics}

We included 70 patients (54 male and 16 female) diagnosed with metastatic clear-cell RCC. The median age of the patients was 62 years (age range between 25 and 79 years old). Most patients had an intermediate prognosis according to MSKCC criteria (41 patients, 58\%), with the rest having poor prognosis (25 patients, $36 \%)$ and a few favourable (4 patients, $6 \%$ ) (Table 1 ).

\section{Response to treatment}

From the 70 patients who participated in the study, 62 were evaluable for response; 50 patients had a clinical benefit $(\mathrm{CB})$ [2 patients had complete response, 24 patients had a partial response (PR) and 24 patients had stable disease (SD)] and 12 patients exhibited disease progression (DP) during or after 2 cycles of treatment and discontinued.

Median progression-free survival (PFS) was 9.47 months whilst median overall survival was 22.03 months (Fig. 1). Median follow-up was 19.2 months.

Following relapse, $\mathrm{m}$-Tor inhibitors, namely temsirolimus and everolimus, have been commenced as salvage therapy.

\section{Thyroid function during treatment}

All patients had an assessment of their thyroid values at baseline. Three patients were diagnosed with subclinical hypothyroidism. 
Table 1 Patient characteristics

\begin{tabular}{lll}
\hline Patient characteristics & Absolute number & $\%$ \\
\hline Number & 70 & 100 \\
Total included & 62 & 88 \\
Evaluable for response & 54 & 77 \\
Sex & 16 & 23 \\
Male & & \\
Female & 62 & \\
Age & $25-79$ & \\
Median & & 43 \\
Range & 30 & 40 \\
Performance Status & 28 & 17 \\
0 & 12 & \\
1 & & 6 \\
2 & 4 & 36 \\
MSKCC risk classification & 41 & \\
Favourable & 25 & \\
Intermediate & Poor &
\end{tabular}

Characteristics of all patients enrolled in the study

All patients received sunitinib treatment and were reassessed for thyroid function 15 days later.

Hypothyroidism arose earlier than expected, since 30 patients $(42.86 \%)$ had a TSH over the upper normal limit, only 15 days after the beginning of sunitinib therapy. At the end of the second cycle (day-75) 35 patients (50.0\%) were reported to have a TSH-increase above the higher normal value. The median TSH concentration at baseline was $1.96 \mathrm{mU} / \mathrm{L}$ and the average TSH concentration was $2.79 \mathrm{mU} / \mathrm{L}$. On day- 15 the median TSH level was 3.88 $\mathrm{mU} / \mathrm{L}$ and the average TSH level was $5.50 \mathrm{mU} / \mathrm{L}$, while on day-75 it was $5.19 \mathrm{mU} / \mathrm{L}$ and $10.04 \mathrm{mU} / \mathrm{L}$ respectively. All free $\mathrm{T}_{4}$ values were determined in the normal range, except one case of a patient that had a TSH concentration of $60.64 \mathrm{mU} / \mathrm{L}$ with a free $\mathrm{T}_{4}$ value of $7.12 \mathrm{pg} / \mathrm{mL}$ (day-75). From the patients who developed hypothyroidism during treatment, only 2 were found with elevated titres of TPOAb, 2 patients with increased TgAb and 1 patient with both antibodies over the normal limit. Moreover, 2 patients developed higher than normal TR-Ab, 1 of whom was diagnosed with hypothyroidism. Depending on the TSH levels in combination with a hypothyroid-related clinical status, levothyroxine was prescribed in order to achieve a TSH value between 0.50 and $2.50 \mathrm{mU} / \mathrm{L}$.

\section{Thyroid function and response to treatment}

On day-15 there were only 8 patients that experienced a decrease in TSH while the majority had increased TSH levels. Mean TSH increase was 2.52 times baseline values (paired t-test $p<0.00001$ ) after 15 days of treatment and 3.68 times baseline values after 75 days of treatment (by end of cycle 2, paired t-test $p<0.00001$ ). TSH increased at an earlier time point (Fig. 2) in patients that exhibited an objective response $(\times 3.33$ times the baseline values on day-15) than patients that exhibited disease stabilisation $(x$ $2.18)$ or disease progression $(\times 1.59)$. These differences were less prominent on day-75 $(3.19,3.41$ and 3.96 respectively]. Overall, these data suggest that early increase in TSH levels may predict clinical benefit from sunitinib in metastatic RCC patients.

\section{Impact of hypothyroidism on survival}

We analysed the association between early increase in TSH levels and progression-free or overall survival. There was a statistically significant association between TSH increase and PFS on first line sunitinib as seen in Fig. 3. Median PFS was 11.92 months for patients that had more than double the baseline TSH levels and 8.63 months for patients that had a day-15/baseline TSH ratio smaller than 2 . The difference was statistically

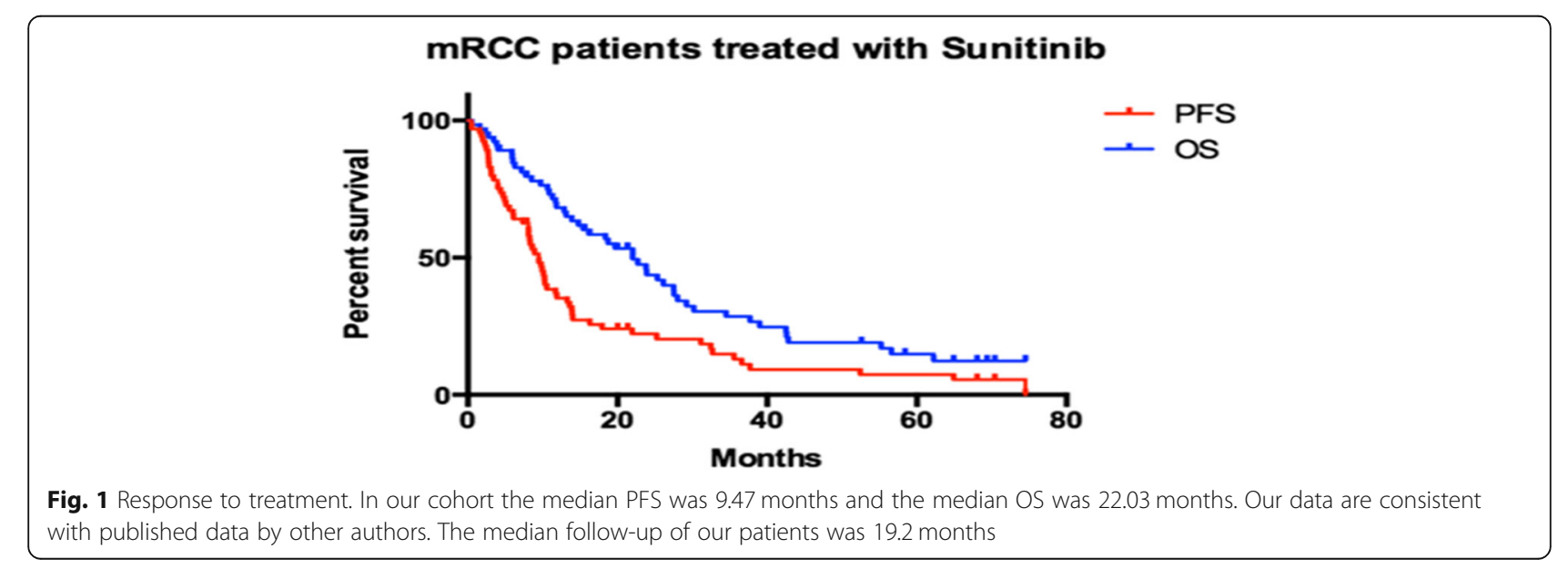




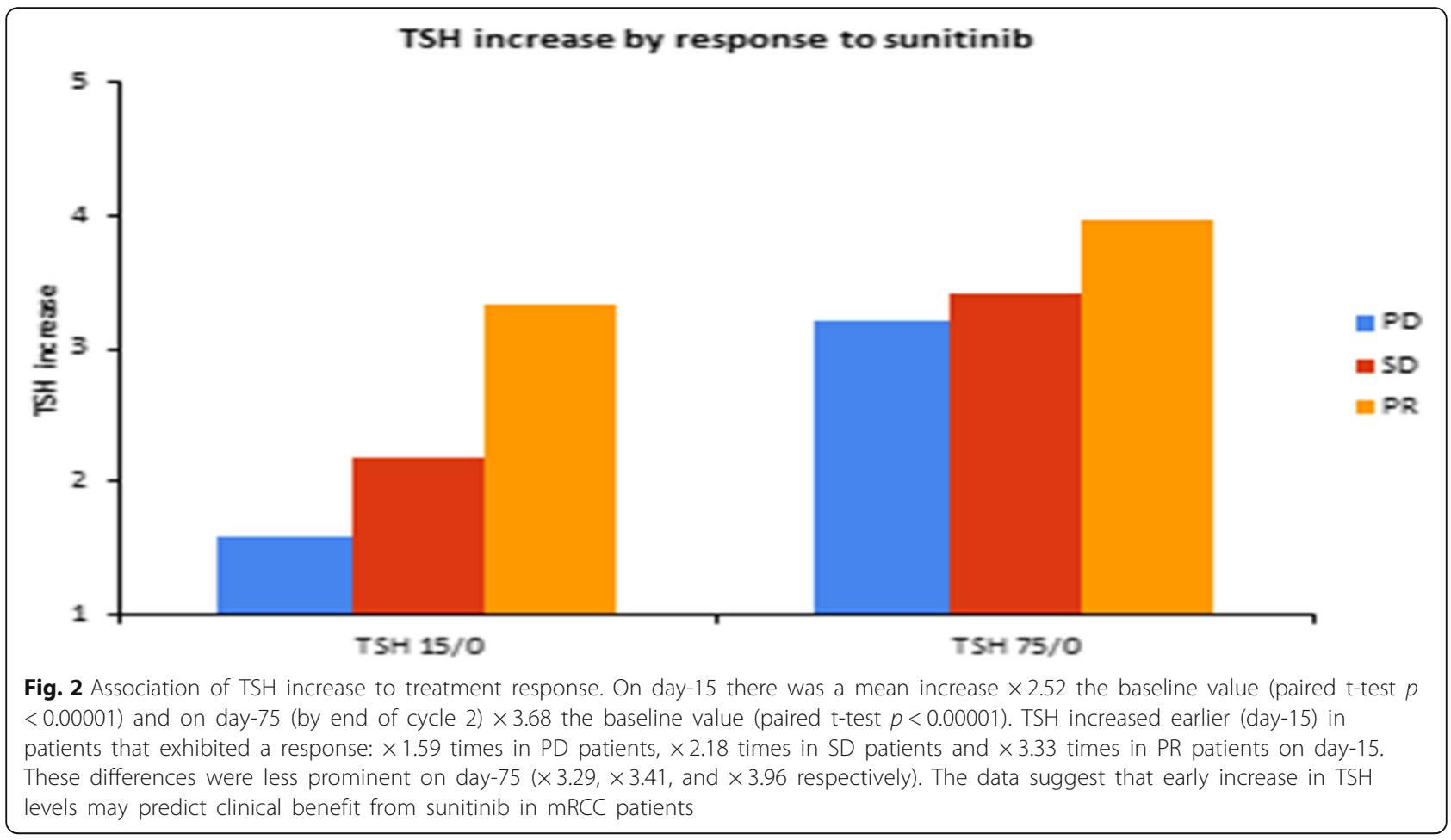

significant with a $p$ value of 0.0476 (log-rank test) and a hazard ratio of 1.76 (95\% CI: 1.033 to 3.288 ). However, early TSH increase had a more prominent impact on overall survival (Fig. 4). Median OS was 3.10 years in patients that had a day-15/baseline $\mathrm{TSH}$ ratio of $>2$ and 1.08 years for those whose ratio was $<2$. The hazard ratio was 1.73 (95\% CI: 1.556 to 5.394 ) and the result was statistically significant with a $p$ value of 0.0011 (log-rank test). There were no prognostic factors that exhibited impact on response other than the established ones such as the MSKCC criteria. However, the impact of early increase of TSH levels had a prognostic effect throughout all MSKCC categories.

\section{Discussion}

The study addresses the following three points: the response of the mRCC patients to the treatment with sunitinib, the manifestation of sunitinib-induced TSH increase and, by extension, the appearance of hypothyroidism and finally the influence of the latter on the patients' survival.

Regarding the response to sunitinib treatment, the results of this study demonstrate a progression-free survival of 9.47 months and an overall survival of 22.03 months, in concert with our previously published results [28].

Hypothyroidism has been reported as a typical and usual side effect of the sunitinib treatment by many writers in both retrospective and prospective studies. In

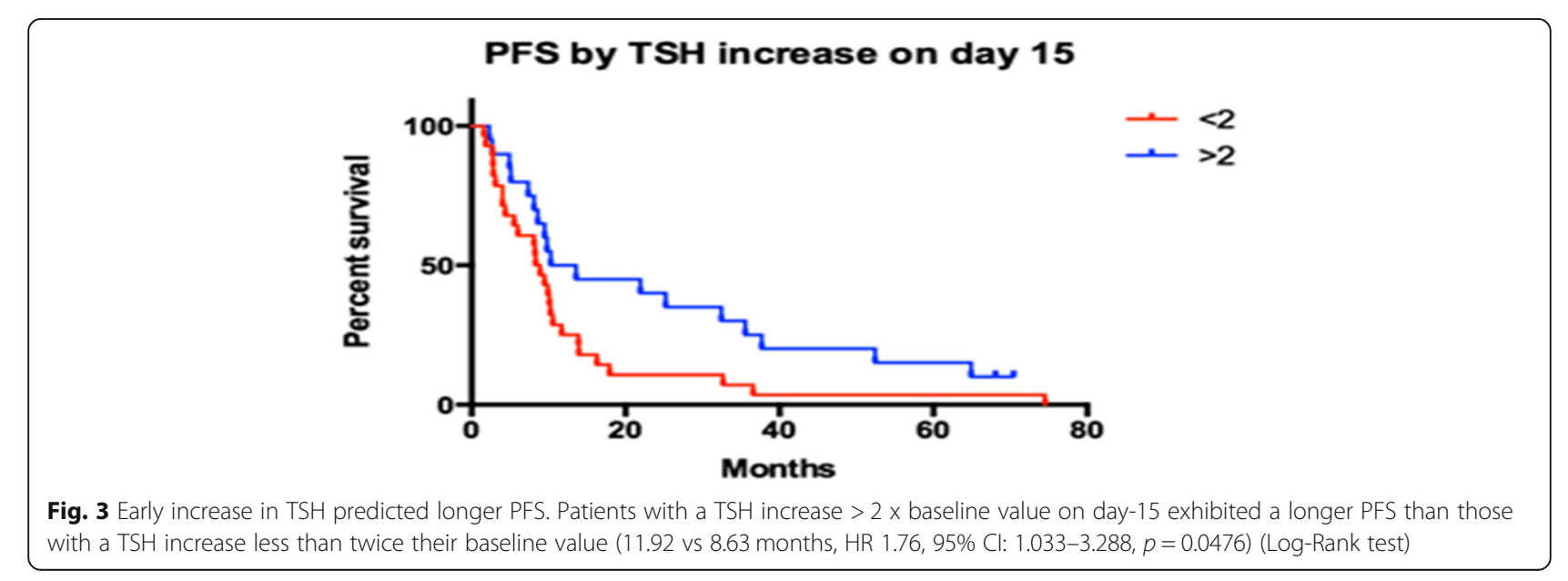




\section{OS by TSH increase on day 15}

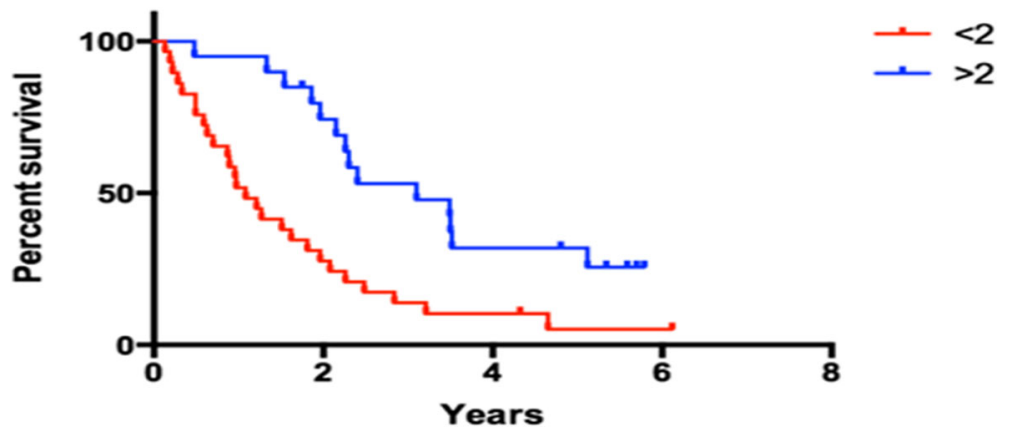

Fig. 4 Early increase in TSH predicted longer OS. Patients with a TSH increase more than twice their baseline value on day-15 exhibited a longer OS than those with a TSH increase less than twice their baseline value (3.1 vs 1.08 years, HR 1.73, 95\% Cl: 1.556-5.394, $p=0.0011$ ) (Log-Rank test). The result is statistically significant

our study, we observed that $42.86 \%$ of the patients were already hypothyroid only 2 weeks after starting sunitinib. Hypothyroid status becomes more common as sunitinib treatment is continued and $50 \%$ of the patients were hypothyroid at the end of the second therapy cycle. The fact that roughly all members of the cohort did not have abnormal thyroid antibody levels, coincides with the conclusion of many studies that autosomal mechanisms do not play a critical role in the emergence of hypothyroidism $[11,12,16,29]$. The physiological free $\mathrm{T}_{4}$ titres in all patients - with the exception of one case - verify the occurrence of subclinical hypothyroidism and the need of thyroid supplementation with levothyroxine depending mainly on the clinical picture.

A considerable number of studies have correlated the presence of hypothyroidism with a longer PFS and OS, as previously reported. In our study, we noticed that early TSH-increase is associated with a clinical benefit. At the end of the second week from the beginning of the treatment, an important number of patients exhibited an elevation of TSH values. The patients that showed at least a twofold increase of their baseline TSH titres responded to therapy by stabilisation or by regression of disease. On the other hand, patients with a less remarkable or non-elevated serum TSH had a higher chance to experience disease progression after two cycles of treatment.

A highly significant observation in our study is that the magnitude of TSH increase in the very early stages of treatment with sunitinib (day-15) appears to have a prognostic value. The PFS of the patients who demonstrated a higher than a twofold rise was significantly longer than the PFS of the patients that presented a smaller or no TSH-increase and this was reflected to overall survival. As the increase in OS is much more prominent than the increase in PFS we have to exclude the hypothesis of a pharmacodynamic or pharmacokinetic effect of sunitinib treatment and rather generate a hypothesis of a "class effect" in thyroid function (as $>30 \%$ of our patients received another RTKI in the 2nd or 3rd line) or a mere effect of hypothyroidism on mRCC (as suggested by Schmidinger et al).

\section{Conclusions}

Our study reports a PFS and an OS for mRCC patients treated with sunitinib which are in concordance with previous studies. Hypothyroidism is a common side effect associated with sunitinib and this study demonstrated a particularly early onset. Hypothyroidism occurred only 2 weeks after commencing sunitinib while its occurrence in the population studied increased further by the end of the second therapy cycle. This early TSH-increase is related to a clinical benefit; specifically, those with at least a twofold increase of their baseline TSH titres responded to therapy by stabilisation or by regression of disease in comparison to those with a smaller or no increase of their baseline TSH who demonstrated a higher chance of disease progression. Finally, both the PFS and the OS of patients who presented a higher than a twofold rise of their baseline TSH on day-15 were significantly longer than the PFS and the OS of patients that exhibited a smaller or no increase of their baseline TSH, with the OS prolongation being more evident.

\section{Abbreviations \\ CB: Clinical benefit; DP: Disease progression; GIST: Gastrointestinal stromal tumour; IFN: Interferon; IRMA: Immunoradiometric assay; mRCC: Metastatic renal cell carcinoma; NIS: Sodium iodide symporter; OS: Overall survival; PFS: Progression-free survival; PR: Partial response; SD: Stable disease; $T_{3}$ : Triiodothyronine; $T_{4}$ : Thyroxine; TgAb: Antibodies against Thyroglobulin; TKIs: Tyrosine kinase inhibitors; TPOAb: Antibodies against thyroid peroxidase; TR-Ab: Antibodies against TSH receptor; TSH: Thyroid - stimulating hormone}

\section{Acknowledgements}

We are obliged to the patients that participated in the study as well as to the staff of the Nuclear Medicine Department at the 'Theagenion' Cancer Hospital for their support in performing the IRMA tests. 


\section{Funding}

No funding was obtained for this study.

\section{Availability of data and materials}

The datasets used and/or analysed during the current study are available from the corresponding author upon reasonable request.

\section{Authors' contributions}

TV designed the study, evaluated the patients, collected data, interpreted the results and prepared the manuscript. MC, KK and MS provided consultation on study design and critically reviewed the manuscript and the interpretation of the data. KP was responsible for the treatment of the patients, collection of data and interpretation of the results and performed the statistical analysis. AS helped with the plasma databank and supervised the performance of the IRMA tests. All authors read and approved the final manuscript.

\section{Ethics approval and consent to participate}

The study and the publication of the relevant produced data were approved - prior to commencing - by the ethics review board of the 'Theagenion' Cancer Hospital (Thessaloniki, Greece). In addition, the study was undertaken in accordance with the Declaration of Helsinki and Good Clinical Practice Guidelines. Finally, a consent form - which included permission for publishing of the collected data - was signed by all patients after being informed.

\section{Consent for publication}

Not Applicable.

\section{Competing interests}

The authors declare that they have no competing interests.

\section{Publisher's Note}

Springer Nature remains neutral with regard to jurisdictional claims in published maps and institutional affiliations.

\section{Author details}

${ }^{1}$ Theagenion Cancer Hospital, Al. Symeonidi 2, 54007 Thessaloniki, Greece. 2Urology Department, Attikon Hospital, Rimini 1, Chaidari, 12462 Athens, Greece. ${ }^{3}$ 2nd Department of Propedeutic Surgery, Laiko Hospital, Agiou Thoma 17, 11527 Athens, Greece. ${ }^{4}$ School of Medicine, National Kapodistrian University of Athens, 15772 Athens, Greece. ${ }^{5}$ Present Address: Endocrinology Department, Hull Royal Infirmary, Anlaby Road, Hull HU3 2JZ, UK. ${ }^{6}$ Present Address: Oncology Department, Geniki Kliniki, M. Kallas 11 Gravias 2, 54645 Thessaloniki, Greece. ${ }^{7}$ Radiation Physics Department, Queen's Centre for Oncology and Haematology, Castle Hill Hospital, Castle Rd, Cottingham HU16 5JQ, UK

\section{Received: 10 July 2017 Accepted: 12 April 2019}

\section{Published online: 30 April 2019}

\section{References}

1. Ferlay J, Steliarova-Foucher E, Lortet-Tieulent J, et al. Cancer incidence and mortality patterns in Europe: estimates for 40 countries in 2012. Eur J Cancer. 2013;49:1374-403.

2. The Surveillance, Epidemiology, and End Results (SEER) Program of the National Cancer Institute. 2014. http://seer.cancer.gov/statfacts/html/kidrp. html\#incidence-mortality. Accessed 31 Jan 2017.

3. Devesa SS, Silverman DT, McLaughlin JK, et al. Comparison of the descriptive epidemiology of urinary tract cancers. Cancer Causes Control. 1990;1:133-41.

4. Yagoda A, Abi-Rached B, Petrylak D. Chemotherapy for advanced renal-cell carcinoma: 1983-1993. Semin Oncol. 1995;22:42-60.

5. Rosenberg SA, Yang JC, Topalian SL, et al. Treatment of 283 consecutive patients with metastatic melanoma or renal cell cancer using high-dose bolus interleukin 2. JAMA. 1994:271:907-13.

6. Bex A, Mallo H, Kerst M, et al. A phase-Il study of pegylated interferon alfa$2 \mathrm{~b}$ for patients with metastatic renal cell carcinoma and removal of the primary tumor. Cancer Immunol Immunother. 2005;54:713-9.

7. Motzer RJ, Hutson TE, Tomczak $P$, et al. Sunitinib versus interferon alfa in metastatic renal-cell carcinoma. N Engl J Med. 2007;356(2):115-24
8. Schmidinger M, Bellmunt J. Plethora of agents, plethora of targets, plethora of side effects in metastatic renal cell carcinoma. Cancer Treat Rev. 2010;36:416-24.

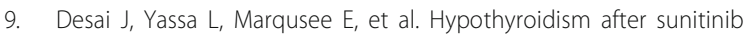
treatment for patients with gastrointestinal stromal tumors. Ann Intern Med. 2006;145:660-4

10. Schoeffski $P$, Wolter $P$, Himpe $U$, et al. Sunitinib-related thyroid dysfunction: a single-center retrospective and prospective evaluation. J Clin Oncol. 2006; 24(Suppl 18):3092.

11. Rini BI, Tamaskar I, Shaheen P, et al. Hypothyroidism in patients with metastatic renal cell carcinoma treated with sunitinib. J Natl Cancer Inst. 2007;99:81-3.

12. Wolter $P$, Stefan $C$, Decallonne B, et al. The clinical implications of sunitinibinduced hypothyroidism: a prospective evaluation. Br J Cancer. 2008;99:448-54

13. Baldazzi $V$, Tassi $R$, Lapini A, et al. The impact of sunitinib-induced hypothyroidism on progression-free survival of metastatic renal cancer patients: a prospective single-center study. Urol Oncol. 2012;30(5):704-10.

14. Wong $E$, Rosen LS, Mulay M, et al. Sunitinib induces hypothyroidism in advanced cancer patients and may inhibit thyroid peroxidase activity. Thyroid. 2007;17(4):351-5.

15. Mannavola D, Coco P, Vannucchi G, et al. A novel tyrosine kinase selective inhibitor, Sunitinib, induces transient hypothyroidism by blocking iodine uptake. J Clin Endocrinol Metab. 2007:92:3531-4.

16. Kappers M, van Esch J, Smedts F, et al. Sunitinib-induced hypothyroidism is due to induction of type 3 deiodinase activity and thyroidal capillary regression. J Clin Endocrinol Metab. 2011;96(10):3087-94.

17. Shinohara N, Takahashi M, Kamishima T, et al. The incidence and mechanism of sunitinib-induced thyroid atrophy in patients with metastatic renal cell carcinoma. Br J Cancer. 2011;104:241-7.

18. Rogiers A, Wolter P, Op de Beeck K, et al. Shrinkage of thyroid volume in sunitinib-treated patients with renal-cell carcinoma: a potential marker of irreversible thyroid dysfunction? Thyroid. 2010;20(3):317-22.

19. Kitajima K, Takahashi S, Maeda T, et al. Thyroid size change by CT monitoring after sorafenib or sunitinib treatment in patients with renal cell carcinoma: comparison with thyroid function. Eur J Radiol. 2012;81:2060-5.

20. Davis FB, Tang HY, Shih A, et al. Acting via a cell surface receptor, thyroid hormone is a growth factor for glioma cells. Cancer Res. 2006;66(14):7270-5.

21. Theodossiou C, Skrepnik N, Robert EG, et al. Propylthiouracil-induced hypothyroidism reduces xenograft tumor growth in athymic nude mice. Cancer. 1999;86(8):1596-601.

22. Goodman AD, Hoekstra SJ, Marsh PS. Effects of hypothyroidism on the induction and growth of mammary cancer induced by 7,12dimethylbenz(a)anthracene in the rat. Cancer Res. 1980:40:2336-42.

23. Schmidinger $M$, Vogl $U$, Bojic $M$, et al. Hypothyroidism in patients with renal cell carcinoma blessing or curse? Cancer. 2011:117(3):534-44

24. Riesenbeck LM, Bierer S, Hoffmeister I, et al. Hypothyroidism correlates with a better prognosis in metastatic renal cancer patients treated with sorafenib or sunitinib. World J Urol. 2011;29:807-13.

25. Sella A, Hercbergs A, et al. Does sunitinib-induced hypothyroidism play a role in the activity of sunitinib in metastatic renal cell carcinoma? Chemotherapy. 2012;58:200-5.

26. Sabatier R, Eymard JC, Walz J, et al. Could thyroid dysfunction influence outcome in sunitinib-treated metastatic renal cell carcinoma? Ann Oncol. 2012;23(3):714-21.

27. Therasse P, Arbuck SG, Eisenhauer EA, et al. New guidelines to evaluate the response to treatment in solid tumors: European Organisation for Research and Treatment of Cancer, National Cancer Institute of the United States, National Cancer Institute of Canada. J Natl Cancer Inst. 2000;92(3):205-16.

28. Kontovinis LF, Papazisis KT, Touplikioti $P$, et al. Sunitinib treatment for patients with clear-cell metastatic renal cell carcinoma: clinical outcomes and plasma angiogenesis markers. BMC Cancer. 2009:9:82.

29. Sato S, Muraishi K, Tani J, et al. Clinical characteristics of thyroid abnormalities induced by sunitinib treatment in Japanese patients with renal cell carcinoma. Endocr J. 2010;57(10):873-80. 